\title{
USING RELATIVE POSITION AND TEMPORAL JUDGMENTS TO ASSESS THE EFFECTS OF TEXTURE AND FIELD OF VIEW ON SPATIAL AWARENESS FOR SYNTHETIC VISION SYSTEMS DISPLAYS
}

\author{
Matthew L. Bolton \\ University of Virginia \\ Charlottesville, Virginia
}

\author{
Ellen J. Bass \\ University of Virginia \\ Charlottesville, Virginia
}

\author{
James R. Comstock, Jr. \\ NASA Langley Research Center \\ Hampton, Virginia
}

Synthetic Vision Systems (SVS) depict computer generated views of terrain surrounding an aircraft. In the assessment of textures and field of view (FOV) for SVS, no studies have directly measured the 3 levels of spatial awareness: identification of terrain, its relative spatial location, and its relative temporal location. This work introduced spatial awareness measures and used them to evaluate texture and FOV in SVS displays. Eighteen pilots made 4 judgments (relative angle, distance, height, and abeam time) regarding the location of terrain points displayed in 112 5-second, non-interactive simulations of a SVS heads down display. Texture produced significant main effects and trends for the magnitude of error in the relative distance, angle, and abeam time judgments. FOV was significant for the directional magnitude of error in the relative distance, angle, and height judgments. Pilots also provided subjective terrain awareness ratings that were compared with the judgment based measures. The study found that elevation fishnet, photo fishnet, and photo elevation fishnet textures best supported spatial awareness for both the judgments and the subjective awareness measures.

\section{INTRODUCTION}

Controlled Flight Into Terrain (CFIT), where a fully functional aircraft is inadvertently flown into the ground, water, or other terrain obstacle, has caused more than $25 \%$ of commercial aviation accidents since 1987 (Boeing, 2005).

CFIT accidents are characterized by a loss of awareness in low level flight and low visibility conditions (FSF, 1999).

Synthetic Vision Systems (SVS) combat this problem. By using onboard terrain and obstacle databases and Global Positioning System data, SVS displays create a synthetic, clear-day view of the world surrounding ownship regardless of visibility (Arthur, et al., 2004).

\section{Spatial Awareness}

Spatial awareness is defined as the extent to which a pilot notices objects in the environment, understands where these objects are with respect to ownship, and understands where these objects will be relative to ownship in the future (Wickens, 2002). This is relevant to SVS since it encompasses a pilot's knowledge about the relative position of terrain.

In SVS and related research, performance measures include cross track error (Alexander, Wickens, \& Hardy, 2003), the number of correct identifications made when matching video of actual terrain to SVS displays (Schnell and Lemos, 2002), reproduction of highlighted terrain points on a blanked SVS Primary Flight Display (PFD) (Alexander et al., 2003), ordinal distance judgments (Yeh, 1992), and azimuth and elevation angle judgments of the relative position of two objects over synthetic terrain (McGreevy \& Ellis, 1986). Subjective awareness measures have also been used in SVS research: Situation Awareness Rating Technique (SART) (Hughes, \& Takallu, 2002), Situation Awareness - Subjective Workload Dominance (SA-SWORD) (Hughes \& Takallu, 2003; Arthur et al., 2004), and terrain awareness (Glaab \& Hughes, 2003).

In these experiments, none of the measures directly probe the pilot's knowledge of all three levels of spatial awareness.

\section{Terrain Texture}

Terrain texture (Figure 1) refers to the imagery drawn on the synthetic terrain of SVS displays. SVS display evaluations have used the following textures (Glaab \& Hughes, 2003):

- Fishnet (F): A grid of interlinked $500 \mathrm{ft}$ by $500 \mathrm{ft}$ squares is drawn on a solidly colored terrain.

- Elevation (E): Distinct bands of color drawn on the terrain represent regularly spaced intervals of terrain elevations which correspond to color schemes used by Visual Flight Rule sectional charts.

- Photo (P): Satellite photos of the actual terrain are superimposed on the synthetic terrain.

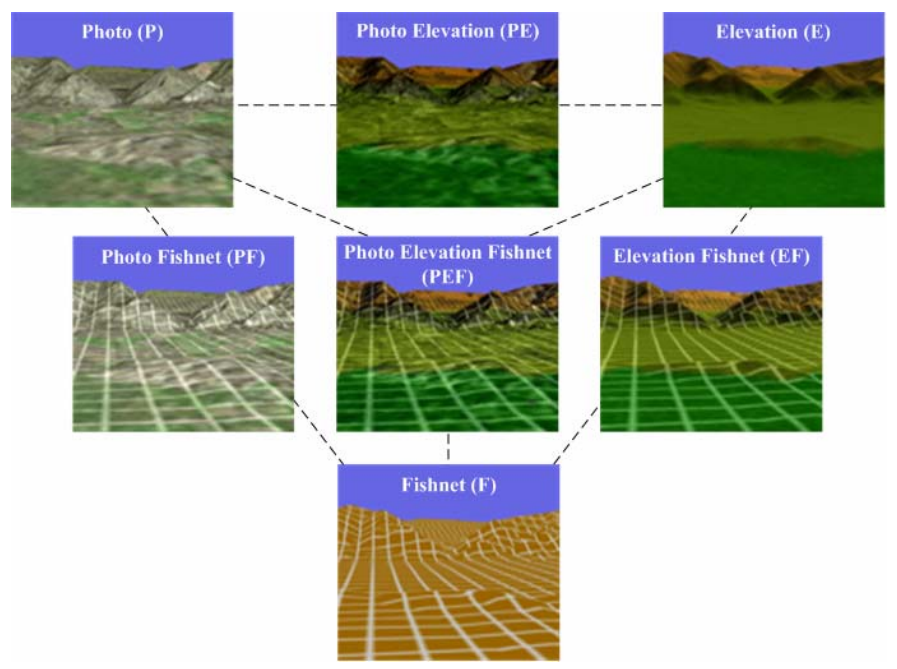

Figure 1. The terrain textures evaluated in the experiment.

Terrain texture is important to spatial awareness because of its ability to facilitate different depth and motion cues. Because SVS displays are 2-d representations of a 3-d space, only pictorial depth cues (cues that can be represented in a 
picture) are relevant. Texture facilitates all of the following pictorial depth cues in SVS displays (Goldstein, 2002):

- Familiar size: When an object's size is known by the observer, he can determine its relative distance by observing how big it is in the 2-d display.

- Relative size: An object that is the same size as another, but is farther away from the observer, will be smaller in the 2-d display.

- Texture gradient: As objects and patterns that are equally spaced get farther away from the observer, they get closer together in the 2-d display.

- Linear Perspective: Parallel lines will converge in the 2-d display as their distance from the observer increases.

The three base texture concepts (fishnet, elevation, and photo) convey different information. The fishnet texture contributes to familiar size, relative size, texture gradient, and linear perspective. Photo texturing provides a more natural view of terrain and contributes to familiar size, relative size, and texture gradient. Elevation texturing provides coded elevation information about the terrain. All textures contribute to the global optic flow motion cue by texturing the invariant structures in the optical ambient array (Gibson, 1986).

In a comparison of six different textures, Schnell and Lemos (2002) found that elevation, photo, and fishnet-like grid textures (a checkerboard pattern) produced the highest percentages of correct identification for video terrain matching and the least amount of cross track error in flight simulator studies. Takallu and Wong (2004) found that photo and elevation texturing produced significantly higher SART scores than no texturing and fishnet texturing. Hughes and Takallu (2003) found that there were significantly larger SA-SWORD scores associated with elevation and photo textures than there were for no texture.

\section{Field of View}

Field of View (FOV) refers to the angular boundaries of the volume of space represented in the display. FOV is important to spatial awareness because in displays with larger FOVs, objects of the same physical size and relative position will be smaller than objects on identically sized displays with smaller FOVs. Experiments conducted by Comstock et al. (2002), Glaab and Hughes (2003), and Arthur et al. (2004) have found that pilots prefer the $30^{\circ} \mathrm{FOV}$ and $60^{\circ} \mathrm{FOV}$.

\section{Objectives and Hypotheses}

The experiment discussed herein investigated the effects of the three leading texture types in all possible combinations (Figure 1) and the two preferred FOVs $\left(30^{\circ}\right.$ and $\left.60^{\circ}\right)$ using spatial awareness measures where participants judged the relative location of a terrain point. Because each of the three base textures conveys different spatial information, it was hypothesized that the highest level of spatial awareness would be achieved by combining all three texture types.

\section{METHODS}

\section{Participants}

Eighteen general aviation pilots participated in the study. All participants had less than 400 hours of flight experience $(\mu$ $=157, \sigma=75$ ). They were familiar with the out the window view from a cockpit but not with SVS displays.

\section{Apparatus}

Experiments were run in a windowless constantly lighted laboratory. Workstations displayed each simulation and collected participant judgments. SVS displays used during simulation were 9.25 in. by 8 in. and used the symbology depicted in Figure 2. In simulations, the location of the terrain point was indicated using a yellow inverted cone $(\mathrm{d}=500 \mathrm{ft}, \mathrm{h}$ $=500 \mathrm{ft}$ ) rendered as part of the SVS environment. The tip of the cone intersected the terrain at the terrain point. All simulations depicted SVS displays in flight at 127 knots. They were displayed as 5 second, $836 \times 728$ pixel, 30 frames per second, Windows Media Video (WMV) files. Custom software played the WMV files and collected participant responses (Bolton, Bass, and Comstock, 2006).

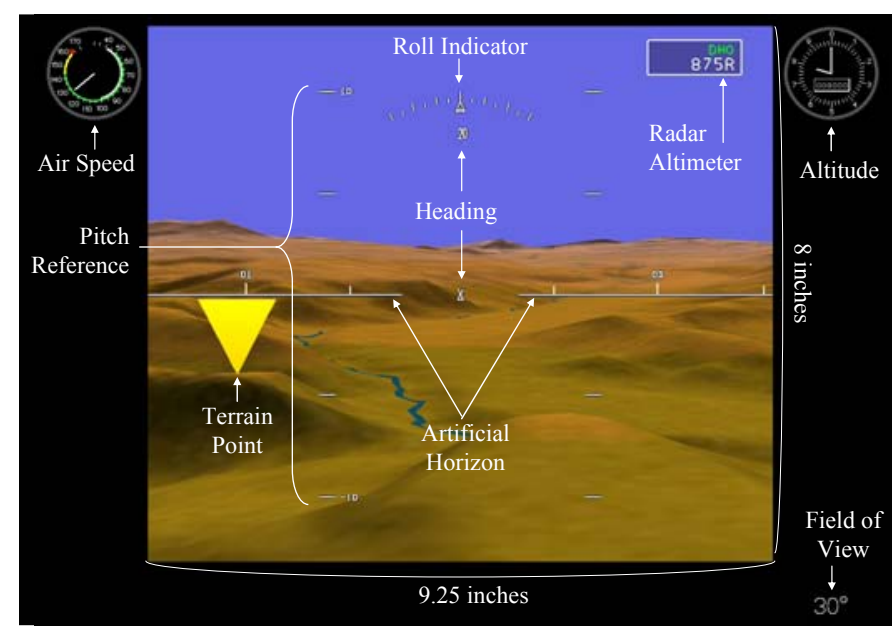

Figure 2. The SVS display used in the experiment.

\section{Independent Variables}

Within Subject Variables. The five within subject variables included texture, FOV, and three for scenario geometry (the relative distance, relative angle, and relative height of the terrain point). Seven textures were used in the experiment: three basic textures (F, P, and $\mathrm{E})$ and four derivative textures (EF, PF, PE, and PEF) (Figure 1). Two FOVs were used in the SVS displays: $30^{\circ}$ and $60^{\circ}$. The location of the terrain point varied based on its relative position at the end of a scenario by changing the three scenario geometry parameters each with two levels (Table 1).

Between Subject Variables. There were two between subject variables: FOV order and texture order. A participant either saw all of the $30^{\circ} \mathrm{FOV}$ trials first or all $60^{\circ} \mathrm{FOV}$ trials first. Thus, FOV order had two levels: $30^{\circ} \mathrm{FOV}$ first or $60^{\circ}$ FOV first. 
Table 1. Terrain Point Relative Position Level Encoding.

\begin{tabular}{llll}
\hline Variable & \multicolumn{1}{c}{ Range } & \multicolumn{1}{c}{ Distribution } & Level \\
\hline Angle & {$\left[0^{\circ}, 6.5^{\circ}\right]$} & $N(\mu=3.75, \sigma=1.25)$ & Small \\
& {$\left[8.5^{\circ}, 15^{\circ}\right]$} & $N(\mu=11.25, \sigma=1.25)$ & Large \\
Distance & {$[1 \mathrm{nmi}, 3.25 \mathrm{nmi}]$} & $N(\mu=2.25, \sigma=0.417)$ & Near \\
& {$[3.75 \mathrm{nmi}, 6 \mathrm{nmi}]$} & $N(\mu=4.75, \sigma=0.417)$ & Far \\
Height & {$[-1000 \mathrm{ft},-100 \mathrm{ft}]$} & $U(-1000,-100)$ & Below \\
& {$[100 \mathrm{ft}, 1000 \mathrm{ft}]$} & $U(100,1000)$ & Above \\
\hline
\end{tabular}

Textures used to derive other textures always appeared before their derivatives. Each participant saw two of the base textures, the combination of them, the third texture, and the rest of the combinations. Three texture orders were created so that no base texture was introduced in more than one ordered slot: $\{\mathrm{P}, \mathrm{E}, \mathrm{PE}, \mathrm{F}, \mathrm{PF}, \mathrm{EF}, \mathrm{PEF}\},\{\mathrm{E}, \mathrm{F}, \mathrm{EF}, \mathrm{P}, \mathrm{PE}, \mathrm{PF}, \mathrm{PEF}\}$, and $\{F, P, P F, E, E F, P E, P E F\}$.

\section{Dependent Variables}

Nine of the 17 dependent variables are discussed in this paper (see Bolton, 2006 for the others). Eight were calculated from the four judgment values (relative angle $\left(^{\circ}\right)$, relative distance (nmi), relative height (ft), and abeam time (s)) from the three judgment tasks (Table 2). There were two dependent variables associated with each judgment value: one for directional error, one for absolute error. Each directional error term represented both the direction and magnitude of the error in the judgment value. When a participant overestimated a judgment, the corresponding directional error term was positive. When the participant underestimated a judgment, it was negative. Absolute error terms represented the magnitude of the error judgment, and were calculated as the absolute value of their corresponding directional error term. Identifying the terrain point probed Level 1 spatial awareness. The relative angle, distance, and height judgments probed Level 2 spatial awareness (the relative location of the terrain). The abeam time judgments probed level 3 spatial awareness (the terrain's relative location in the future).

For each texture and FOV combination, participants provided a subjective terrain awareness score (Awareness) by specifying how aware they were of the relative location of terrain on a one-hundred point scale.

Table 2. Dependent Variable Judgment Error Formulations.

\begin{tabular}{lcccc}
\hline Variable & $\begin{array}{c}\text { Actual } \\
\text { Value }\end{array}$ & $\begin{array}{c}\text { Judgment } \\
\text { Value }\end{array}$ & $\begin{array}{c}\text { Directional } \\
\text { Error }\end{array}$ & $\begin{array}{c}\text { Absolute } \\
\text { Error }\end{array}$ \\
\hline Angle & $A_{a}$ & $A_{j}$ & $A_{e}=\left\{\begin{array}{lll}A_{j}-A_{a} \text { if } A_{a}>0 \\
A_{a}-A_{j} \text { otherwise }\end{array}\right.$ & $\left|A_{e}\right|$ \\
Distance & $D_{a}$ & $D_{j}$ & $D_{e}=D_{j}-D_{a}$ & $\left|D_{e}\right|$ \\
Height & $H_{a}$ & $H_{j}$ & $H_{e}=\left\{\begin{array}{lll}H_{j}-H_{a} \text { if } H_{a}>0 \\
H_{a}-H_{j} \text { otherwise }\end{array}\right.$ & $\left|H_{e}\right|$ \\
Time & $\tau_{a}$ & $\tau_{j}$ & $\tau_{e}=\tau_{j}-\tau_{a}$ & $\left|\tau_{e}\right|$ \\
\hline
\end{tabular}

\section{Procedure}

The participants completed consent forms and were briefed about the experiment. Each was randomly assigned to a workstation and experimental condition. Each viewed five second simulations of an SVS heads down display in flight (Figure 2). At the end of the five seconds, the simulation paused for one second, and the screen was cleared. For each trial, participants made four judgments based on the relative position of the terrain point: relative angle, relative distance, relative height, and abeam time using the interface in Figure 3. For training trials, participants were given feedback relating to the accuracy of their judgments. Participants were asked to perform these tasks as quickly and accurately as possible.

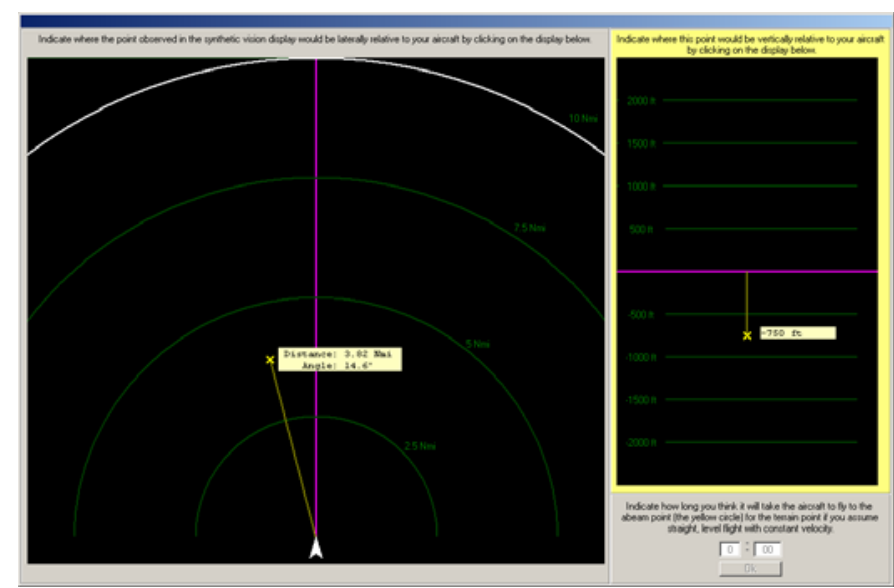

Figure 3. The judgment collection interface.

All participants experienced 112 counterbalanced experimental trials ( 7 textures * 2 FOVs $* 2$ Relative Angles * 2 Relative Distances $* 2$ Relative Heights $=112$ ) and 72 training trials. For the first texture experienced for each FOV, there were 12 training trials. For the other textures, for each FOV, there were 4 training trials. Thus, each participant saw a total of 72 training trials, resulting in a total of 184 trials.

On completion of all of the trials for each texture for each FOV, subjective Demand (where participants assessed how the display configuration placed demand on attentional resources), Awareness, and Clutter (where participants assessed how cluttered each configuration was) ratings were collected using 100 point Likert scales. After all of the trials for a FOV were completed, participants made SA-SWORD pair-wise comparisons between each texture seen with that FOV. After all of the trials were complete, participants indicated which FOV provided the best terrain awareness for each texture (Bolton, 2006).

\section{Experimental Design and Data Analysis}

The experiment employed a repeated measure design with eighteen participants. Three participants were randomly assigned to each of the six combinations of the between subject variables $(2 \mathrm{FOV}$ orders $* 3$ texture orders $=6$ ).

The main and two-way interaction effects of the within and between subject factors on the dependent variables were assessed using a univariate repeated measures analyses of variance (ANOVA) with a Type III sum of squares (Brace, Kemp, and Snelgar, 2003). A Mauchly's Test of Sphericity was performed in order to ensure that the assumptions for the 
repeated measure analysis were not violated. When sphericity was violated $(p<0.05)$ a Greenhouse-Geisser Epsilon correction factor was used (Brace, Kemp, and Snelgar, 2003).

\section{RESULTS}

This section presents the results of the main effects using $\alpha=0.05$ for significance and $\alpha=0.1$ for trends. See Bolton (2006) for more results.

Table 3. Significance and Trends in Main Effects.

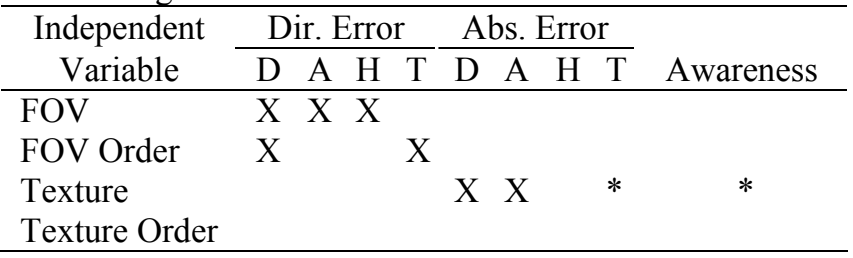

Note. $\mathrm{D}=$ Distance, $\mathrm{A}=$ Angle, $\mathrm{H}=$ Height, $\mathrm{T}=$ Time.

$\mathrm{X} \mathrm{p}<0.05{ }^{*} \mathrm{p}<0.10$.

\section{Texture}

Texture was a significant main effect for absolute distance error and absolute height error. There was a trend for absolute time error. Since texture did not violate sphericity for Absolute Distance Error and Absolute Height Error, the Tukey multiple comparison method (MCM) was used (Stevens 2002). Since texture did violate sphericity for Absolute Time Error, the Bonferroni MCM was used (Stevens 2002). These post hoc analyses revealed that there were three textures that were among the set of textures that produced the smallest average error magnitudes for which there were no trends in differences: EF, PF, and PEF (Table 4).

Texture showed a trend for awareness. While a Tukey's post hoc analysis indicated that there were no significant differences between the mean awareness ratings for each texture, differences were found using a least significant difference MCM. This analysis revealed that EF, PF, and PEF were amongst the four textures that received the highest average awareness ratings, with no trends between ratings (Table 4).

Table 4. Textures that produced the most desirable values (least error and highest awareness ratings) when texture was significant or a trend.

\begin{tabular}{lcccc} 
& \multicolumn{3}{c}{ Absolute Error } & \\
\cline { 2 - 4 } Texture & Angle & Distance & Time & Awareness \\
\hline F & X & & X & \\
E & & & & X \\
P & & X & X & \\
EF & $\mathbf{X}$ & $\mathbf{X}$ & $\mathbf{X}$ & $\mathbf{X}$ \\
PF & $\mathbf{X}$ & $\mathbf{X}$ & $\mathbf{X}$ & $\mathbf{X}$ \\
PE & $\mathbf{X}$ & $\mathbf{X}$ & & \\
PEF & $\mathbf{X}$ & $\mathbf{X}$ & $\mathbf{X}$ & $\mathbf{X}$ \\
\hline
\end{tabular}

FOV

FOV was not significant for any of the absolute error terms. It was significant for Directional Distance, Angle, and
Height Error. Participants tended to underestimate distance judgments with the $30^{\circ} \mathrm{FOV}$, and overestimate distance for the $60^{\circ} \mathrm{FOV}$. They tended to overestimate angle judgments for both FOVs, but more for the $30^{\circ} \mathrm{FOV}$. They also tended to underestimate relative height judgments for both FOVs, but more for the $60^{\circ} \mathrm{FOV}$.

\section{Texture Order}

There was no significant main effect for Texture Order for any of the absolute error terms (Table 3 ). Thus, there is no evidence to suggest that the low error rates observed for the $\mathrm{PF}, \mathrm{EF}$, and PEF textures were dependent on texture order.

\section{FOV Order}

FOV order, was significant or a trend for several error terms (Table 3 ). Those who saw the $30^{\circ} \mathrm{FOV}$, on average, committed significantly less height error than participants who saw the $60^{\circ} \mathrm{FOV}$ first. Those who saw the $30^{\circ} \mathrm{FOV}$ first tended to overestimate relative distances and abeam times, while participants who saw the $60^{\circ} \mathrm{FOV}$ first tended to underestimate them.

\section{DISCUSSION}

The primary objective of this experiment was to determine how different textures and FOVs facilitate spatial awareness in SVS displays.

\section{Texture}

Because the three base textures used in this experiment (Fishnet (F), Elevation (E), and Photo (P)) conveyed different spatial information, this research hypothesized that their combinations would result in increased spatial awareness. For the absolute error terms for which texture was significant or a trend (Absolute Angle, Absolute Distance, and Absolute Time Error), the data do suggest that combinations of the base texture concepts enhance spatial awareness. In all three cases, the PF, EF, and PEF textures were in the set of textures that produced the smallest magnitudes in error and received the highest average awareness ratings (Table 4). Thus, the data suggest that spatial awareness would be best facilitated by one of these three textures.

If only the three base textures $(\mathrm{F}, \mathrm{E}$, and $\mathrm{P})$ are considered, there is no clear winner as each held advantages over the other: $\mathrm{F}$ reduced error in angle judgment, $\mathrm{P}$ reduced error in distance judgment, and E received higher awareness ratings. In order to make a distinction between these textures, the relative importance of each of the dependent measures must be defined.

\section{FOV}

FOV was not a significant main effect for any of the absolute error dependent variables (only for directional error variables). Neither FOV provided superior spatial awareness for all of the relevant directional error variables. Thus, a FOV 
can not be chosen using the results of this study without a method for assessing the relative importance of directional error.

SVS studies by Comstock et al. (2002) and Glaab and Hughes (2003) allowed participants to choose which FOV they used during simulations and flight tests. The data collected in this experiment suggests that such a feature could be problematic if implemented in an actual cockpit. This is predominantly due to the fact that FOV Order had significant effects for Absolute Height Error, Directional Distance Error, and Directional Time Error (Table 3). However, the nature of the experiment (five second, non-interactive flight segments) should suggest that more investigation is warranted.

\section{Conclusions and Future Work}

This work introduced new measures of spatial awareness which were used to evaluate textures and FOVs for SVS displays. It found that spatial awareness was best supported by the EF, PF, or PEF textures. It also found that a $30^{\circ}$ or $60^{\circ}$ FOV could be used, where a $30^{\circ} \mathrm{FOV}$ would reduce the underestimation of relative heights, and a $60^{\circ} \mathrm{FOV}$ would reduce the overestimation of relative angles.

There are limited generalizations that can be drawn from this study given the artificiality of its procedure: scenarios were short and independent of each other, the in flight segments were non-interactive, and the terrain point was indicated using an unrealistic object. Thus, this procedure could potentially be improved by incorporating the spatial awareness judgments into more realistic flight scenarios (where cross track error could be collected) using more realistic terrain point indicators (runways, towers, etc.).

There are also a variety of other display parameters and pictorial depth cues that could affect pilot spatial awareness in SVS. Such display parameters include additional FOVs, additional textures (see Schnell and Lemos, 2002), and different display sizes (see Comstock et al. 2002). There are also depth cues that could be evaluated. Objects familiar to pilots, such as buildings, trees, and towers could be added to the SVS display to facilitate relative size and familiar size depth cues. Since the three best textures from this experiment (EF, PF, and PEF) both contained grid patterns, research could investigate what grid sizes result the most accurate spatial judgments. Atmospheric perspective is a depth cue where an object that is farther away appears less sharp than an object that is closer due to dust, moisture, and other particulates in the air (Goldstein, 2002). This depth cue could be added to SVS displays so that its impact on spatial awareness could be evaluated.

\section{ACKNOWLEGEMENTS}

This work was supported in part by the NIA and NASA. The authors would like to thank those making suggestions for this study including: Trey Arthur, Daniel Burdette, Sheila
Conway, Stephanie Guerlain, Regina Johns, Lynda Kramer, Kara Latorella, Neil O'Connor, Hayes Press, Valerie Plyler, Lawrence Prinzel, Paul Schutte, Jason Sweeters, Mohammad Takallu, and Steve Williams.

\section{REFERENCES}

Alexander, A. L., Wickens, C., \& Hardy, T. J. (2003). Examining the effects of guidance symbology, display size, and field of view on flight performance and situation awareness. Proceedings of the Human Factors and Ergonomics Society $47^{\text {th }}$ Annual Meeting. Denver, CO.

Arthur, J. J., Prinzel, L. J., III, Kramer, L. J., Parrish, R. V., \& Bailey, R. E. (2004) Flight simulator evaluation of synthetic vision display concepts to prevent controlled flight into terrain (CFIT). NASA Technical Report NASA/TP-2004-213008.

Boeing Commercial Airplanes. (2005, May). Statistical summary of commercial jet airplane accidents: World wide operations $1959-2004$. Aviation Safety. Retrieved August 1, 2005 from World Wide Web: http://www.boeing.com/news/techissues/pdf/statsum.pdf

Bolton, M. (2006). An Investigation of the Effects of Texture and Field of View on Spatial Awareness in Synthetic Vision Head-down Displays. Unpublished master's thesis, University of Virginia, Charlottesville, VA.

Bolton, M. L., Bass, E. J., \& Comstock, J. R. (2006). A toolset to support the development of spatial and temporal judgment experiments for synthetic vision systems. 2006 IEEE Systems and Information Engineering Design Symposium. April 28, 2006, Charlottesville, VA.

Brace, N., Kemp, R., \& Snelgar, R. (2003) SPSS for psychologists: A guide to data analysis using SPSS for Windows. Mahwah, NJ: Lawrence Erlbaum Associates, Inc.

Comstock, J. R., Glaab, L. J., Prinzel, L. J., \& Elliott, D. M. (2002). Can effective synthetic vision system displays be implemented on limited size display spaces? Proceedings of the $11^{\text {th }}$ International Symposium on Aviation Psychology. Dayton, Ohio: The Ohio State University.

Flight Safety Foundation. (1999). Killers in aviation: LFSF task force presents facts about approach-and-landing and controlled-flight-into-terrain accidents. Eurocontrol. Retrieved August 1, 2005.

Gibson, J. J. (1986). The ecological approach to visual perception. Hillsdale: Lawrence Erlbaum Associates.

Glaab, L. J., \& Hughes, M. F. (2003). Terrain portrayal for head-down displays flight test. Proceedings of the $22^{\text {nd }}$ Digital Avionics Systems Conference. Indianapolis, Indiana.

Goldstein, E. B. (2002). Sensation and perception. $6^{\text {th }}$ ed. Pacific Grove: Wadsworth Group.

Hughes, M. F. \& Takallu, M. A. (2002). Terrain portrayal for head-down displays experiment. Proceedings of the International Advanced Aviation Conference.

McGreevy, M. W. \& Ellis, S. R. (1986). The effect of perspective geometry on judged direction in spatial information instruments. Human Factors, 28(4), 439-456.

Schnell, T. \& Lemos, K. (2002). Terrain sampling density and texture requirements for synthetic vision systems. Rockwell Collins Final Report. Cedar Rapids, IA: Rockwell Collins.

Stevens, J. P. (2002) Applied multivariate statistics for the social sciences. Mahwah, NJ: Lawrence Erlbaum Associates, Inc.

Takallu, M. A., Wong, D.T., Bartolone, A.P., Hughes, M.F., \& Glaab, L.J. (2004). Interaction between various terrain portrayals and guidance/tunnel symbology concepts for general aviation synthetic vision displays during a low en-route scenario. Proceeds of the 2004 Digital Avionics Systems Conference (DASC). Salt Lake City, UT.

Wickens, C. D. (2002). Spatial awareness biases. NASA Technical Report ARL-02-6/NASA-02-4.

Yeh, Y. (1992). Spatial judgments with monoscopic and stereoscopic presentation of perspective displays. Human Factors, 34(5), 583-600. 